\title{
Macranthoidin B Modulates Key Metabolic Pathways to Enhance ROS Generation and Induce Cytotoxicity and Apoptosis in Colorectal Cancer
}

\author{
Xing Fan ${ }^{a} \quad$ Jun Rao ${ }^{b}$ Ziwei Zhang ${ }^{c}$ Dengfeng Lic Wenhao Cuid Jun Zhange \\ Hua Wang $^{\mathrm{b}}$ Fangfang Tou ${ }^{\mathrm{b}}$ Zhi Zheng ${ }^{\mathrm{b}, \mathrm{c}}$ Qiang Shen ${ }^{\mathrm{c}}$ \\ aDepartment of Hematology, Shanghai Institute of Hematology, Ruijin Hospital, Shanghai Jiaotong \\ University School of Medicine, Shanghai, ${ }^{b}$ Department of Internal Medicine $5^{\text {th }}$ Division, Jiangxi \\ Cancer Hospital, Jiangxi Cancer Center, Nanchang, China; 'Department of Clinical Cancer Prevention, \\ eDepartment of Hematopathology, The University of Texas MD Anderson Cancer Center, Houston, TX, \\ U.S.A; dDepartment of Cellular Therapy, You Jia Medical Technology Co., Ltd, Shanghai, China
}

\section{Key Words}

Colorectal cancer • Macranthoidin B • Natural product • Metabolomics • Cancer therapy

\begin{abstract}
Background/Aims: Induction of oxidative stress and reactive oxygen species (ROS) mediatedapoptosis have been utilized as effective strategies in anticancer therapy. Macranthoidin B (MB) is a potent inducer of ROS-mediated apoptosis in cancer, but its mechanism of action is poorly understood. Method: Superoxide production with MB exposure in colorectal cancer (CRC) cells was measured using lucigenin chemiluminescence and real-time PCR. MB's inhibitory effect on proliferation and viability of CRC cells was determined by proliferation assays. MB's effect on apoptosis of CRC cells was determined by Western blotting and annexin V-FITC/PI staining. $M B$ 's effect on the growth of CRC xenografts in mice was assessed. An established metabolomics profiling platform combining ultra-performance liquid chromatography-tandem mass spectrometry (LC-MS) with gas chromatography-mass spectrometry (GC-MS) was performed to determine MB's effect on total metabolite variation in CRC cells. Results: We found that MB increases ROS generation via modulating key metabolic pathways. Using metabolomics profiling platform combining LC-MS with GC-MS, a total of 236 metabolites were identified in HCT-116 cells in which 31 metabolites were determined to be significantly regulated ( $p \leq$ 0.05 ) after $\mathrm{MB}$ exposure. A number of key metabolites revealed by metabolomics analysis include glucose, fructose, citrate, arginine, phenylalanine, and S-adenosylhomocysteine (SAH), suggesting specific modulation of metabolism on carbohydrates, amino acids and peptides, lipids, nucleotide, cofactors and vitamins in HCT-116 CRC cells with MB treatment highly associated with apoptosis triggered by enhanced ROS and activated caspase-3. Conclusion: Our results demonstrate that MB represses CRC cell proliferation by inducing ROS-mediated apoptosis.

X. Fan, J. Rao and Z. Zhang contributed equally to this work.




\section{Cellular Physiology Cell Physiol Biochem 2018;46:1317-1330 \\ \begin{tabular}{c|c} 
DOI: 10.1159/000489147 & O 2018 The Author(s). Published by S. Karger AG, Basel \\
www.karger.com/cpb
\end{tabular}}

Fan et al.: Macranthoidin B Modulates Key Metabolic Pathways in Colorectal Cancer

\section{Introduction}

Reactive oxygen species (ROS) are primarily produced by mitochondria and act as secondary messengers in various cellular processes such as cellular proliferation, differentiation, protein synthesis, glucose metabolism, cell survival, and inflammation $[1,2]$. Most cancer cells exhibit increased levels of ROS and oxidative stress, which contribute to cancer development and progression. On the other hand, cancer cells also produce more antioxidant proteins to detoxify the effect from ROS, demonstrating that a delicate and dynamic balance of intracellular ROS level is required for cancer cells [2]. ROS appears a double-edged sword in cancer cells, since modestly increased ROS contributes to sustained cancer cell proliferation and survival, DNA damage and genetic instability, metabolic adaptations, autophagy and drug resistance, whereas excessive ROS production lead to cell death. The ability of cancer cells to distinguish between ROS as a survival or an apoptotic signal is largely controlled by the dosage, duration, type, and site of ROS production [3]. Thus, it was proposed to target the unique biochemical alterations in cancer cells to achieve therapeutic efficacy and prevent the development of drug resistance [4, 5]. In fact, most of the currently available chemotherapeutic and radiotherapeutic agents kill cancer cells by augmenting ROS stress $[6,7]$. In reality, it is plausible to significantly increase intracellular ROS to kill cancer cells by decreasing their antioxidant capacity, by using compounds that inhibit antioxidant systems or through inhibiting specific signaling pathways that upregulate antioxidants in cancer cells. The resulting enhanced ROS in turn induces tumor cell death through either ROS no-specific damaging or by specific induction of apoptosis via death signaling pathways [8]. In this regard, a fine tuning of intracellular ROS signaling may effectively deprive cells from ROS-induced tumor promoting events, instead re-direct the balance to ROS-induced apoptotic signaling in cancer cells [4, 9].

Traditional Chinese medicines (TCMs) have been used for over four thousand years in China's history, and are currently getting more and more attention for their reliable therapeutic efficacy in treating cancers and other diseases. Particularly, the award of the Nobel Prize in 2015 to China's pharmacologist, Youyou Tu who was predominantly responsible for the discovery of artemisin, highlighted an enthusiastic attention towards TCMs and their potential applications in treating and preventing cancer and other diseases [10]. TCMs, used as powerful antioxidants, have been historically documented for maintaining the general health by reducing the risk of chronic diseases including cancers, as reviewed by Ma et al. [11]. In fact, TCMs have unique advantages in preventing and treating cancer when compared with currently used single-molecule "modern" medications, by playing crucial roles in preventing cancer development, attenuating toxicity, enhancing therapeutic efficacy, and reducing tumor recurrence and metastasis [12].

Metabolomics analysis allows comprehensive characterization of global metabolites in specific biological systems, covering key compounds such as amino acids, carbohydrates, lipids, nucleic acids, and so on $[13,14]$. More importantly, this strategy can be used as a powerful tool to manifest the function of organisms from terminal symptoms of metabolic network and understand metabolic changes in response to endogenous and exogenous factors [15]. Metabolomics analysis has been increasingly used to reveal the underlying mechanisms of action for evidence-based Chinese medicine including Chinese medicine syndromes "Zheng", action of Chinese medicine, preventive treatment, Chinese medical formulae, and acupuncture efficacy [15]. This methodology also has the potential for disease diagnosis, screening of new drugs, evaluation of the therapeutic efficacy, safety monitoring, and discovery of new biomarkers of therapeutics or TCM products $[16,17]$. For example, Raina et al. [17] reported a metabolomics study utilizing quantitative high-resolution nuclear magnetic resonance spectroscopy $\left({ }^{1} \mathrm{H}-,{ }^{13} \mathrm{C}\right.$ - and $\left.{ }^{31} \mathrm{P}-\mathrm{NMR}\right)$ to profile the metabolism and energy state of silibinin-treated colorectal cancer (CRC) cells in complementing accompanying proteomics analysis and associated apoptosis in response to silibinininduced energy restriction. Their results suggest that silibinin may dually induce apoptosis and autophagy by restricting glucose uptake and energy production to inhibit CRC cells. 
Moreover, our recent metabolomics study on peiminine, a compound extracted from the bulbs of Fritillaria thunbergii which has been traditionally used in TCM, demonstrated inhibitory effect on CRC cell proliferation by inducing apoptosis and autophagy and modulating key metabolic pathways [14]. In addition, Cui et al. [18] recently reported using LC-MS- and GC-MS-based untargeted metabolomics strategy to quantitatively evaluate the effects of Huangqin decoction on irinotecan-induced gastrointestinal toxicity, suggesting a potential application of metabolomics analysis in elucidating the comprehensive mechanisms of action of TCMs or combinations of medications.

Macranthoidin B (MB) (Fig. 1A) is a triterpenoid saponin extracted from Flos Lonicerae (Shanyinhua in Chinese), which has traditionally been used for centuries as herbal remedy for acute fever, headache, pharyngodynia, respiratory infection, pyocutaneous disease, and epidemic diseases at early stage [19-21]. In recent years, studies revealed that the extraction of saponins from Flos Lonicerae possesses protective effects on hepatic injury caused by acetaminophen and Chemokine (C-C motif) Ligand $4\left(\mathrm{CCl}_{4}\right)[22,23]$. However, there are limited studies reporting MB's anti-cancer effect and impact on reactive oxygen species (ROS). In this study, we attempted to demonstrate the effect of MB exposure on a typical CRC cell line, HCT-116, by inducing ROS generation via modulating key metabolic pathways. We also found significant inhibition of proliferation in cultured CRC cells and suppressed xenograft tumor growth in mice by MB. We further found that MB treatment significantly induces apoptosis by increasing superoxide and decreasing the MnSOD and GSH-Px. Taken together, our study demonstrates that MB induces ROS production, resulting in enhanced ROS-mediated apoptosis and suppressed CRC xenograft tumor growth. These findings provide a rationale for further therapeutic development of this natural product.

\section{Materials and Methods}

\section{Cell culture}

HCT-116 cell line was obtained from ATCC (Manassas, VA). The cells were cultured in Dulbecco's modified Eagle's medium supplemented with $10 \%$ fetal bovine serum in a humidified incubator at $37^{\circ} \mathrm{C}$ in an atmosphere of $5 \% \mathrm{CO}_{2}$. The cells were subcultured when they reached $90 \%$ confluence.

\section{Cell viability assay}

HCT-116 cells were seeded in a 96-well plate (2000 cells/well) in Dulbecco's modified Eagle's medium supplemented with 2\% fetal bovine serum. MB solution (Catalog \# 111814-201604; National Institutes for Food and Drug Control, Beijing, P.R. China) was applied to the cells at final concentrations of 20, 50, 100, 200 , and $400 \mu \mathrm{M}$. To determine cell viability, $48 \mathrm{~h}$ after treatment, $10 \mu \mathrm{L}$ of the ready-to-use reagent CCK8 (catalog \# DB884; Dojindo, Kumamoto, Japan) was added to each well. The cells were then incubated for approximately $30 \mathrm{~min}$ at $37^{\circ} \mathrm{C}$ in a humidified, $5 \% \mathrm{CO}_{2}$ atmosphere. Within $1 \mathrm{~h}$ of completion of the incubation period, absorbance of the formazan dye produced was measured at a wavelength of $450 \mathrm{~nm}$ using a microplate spectrometer. The measured absorbance correlated directly to the number of viable cells.

\section{Western blot}

Total cell lysate was collected and electrophoresis in 4-12\% bis-acrylamide gel with 100V for 2 hours. Proteins were transfer to nitrocellulose membrane with $300 \mathrm{~mA}$ current for 90 minutes. Primary antibodies used for Western blot include Caspase-3 (CST, \#3195), GAPDH (Abcam, ab8245), and TCF8/ZEB1 (CST, \#3396).

\section{Real-time PCR}

Total RNA is isolated using RNeasy Mini Kit (Qiagen) and cDNA was prepared using SuperScript VILO ${ }^{\mathrm{TM}}$ cDNA Synthesis Kit (Invitrogen Corp., Carlsbad, CA). Real-time PCR was performed using the 7300 Real Time PCR System (Applied Biosystems, Foster City, CA) with the SYBR Premix Ex Taq (Takara Bio Inc., Shiga, Japan) ( PMID:22850540). Primers used for real-time PCR was as below: MnSOD sense: TGACCACCACCATTGAACTT; MnSOD antisense: CGTCACCGAGGAGAAGTACC; SH-Px2 sense: TTTTGGACAAGGGTGAAGGT; GSH-Px2 antisense: TGCAACCAATTTGGACATCA. 


\section{Cellular Physiology Cell Physiol Biochem 2018;46:1317-1330 \begin{tabular}{l|l} 
DOI: 10.1159/000489147 & Ond Biochemistry \\
Published online: April 23, 2018 & $\begin{array}{l}\text { 2018 The Author(s). Published by S. Karger AG, Basel } \\
\text { www.karger.com/cpb }\end{array}$
\end{tabular}}

Fan et al.: Macranthoidin B Modulates Key Metabolic Pathways in Colorectal Cancer

\section{Detection of ROS production}

Superoxide production was measured using lucigenin chemiluminescence method described previously [24], and the emission and intensity is shown as relative light units (RLU) per milligram of protein per minute.

\section{Flow cytometry}

HCT-116 cells were treated with $200 \mu \mathrm{M}$ of MB or dimethyl sulfoxide (control) and cultured in Dulbecco's modified Eagle's medium supplemented with 10\% fetal bovine serum for $24 \mathrm{~h}$. The cells were collected and stained with propidium iodide and annexin V (BD Biosciences, Franklin Lakes, NJ) according to the manufacturer's guidelines and analyzed using a BD Influx flow cytometer (BD Biosciences).

\section{Assessment of the effect of $M B$ on xenograft tumor growth in vivo}

To investigate the antitumor activity of $\mathrm{MB}$ in vivo, four-week nude mice, half males and half females, were injected with human CRC cells, HCT-116, and then administrated with MB or vehicle control through intraperitoneal injection respectively. Tumor volume was monitored every 7 days one week post cancer cell injection. Three week post cell injection, mice were euthanized and tumor tissues were removed and weighted.

\section{Metabolite profiling and identification}

Metabolite profiling of HCT-116 cells for MB-treated and MB-untreated group was performed using an established metabolomic profiling platform combining UPLC/LC-MS with GC-MS as previously described $[14,25,26]$. Briefly, samples were prepared with a recovery standard for QC purposes. After extraction, the samples were divided into five fractions including one for analysis by GC-MS, one for analysis by UPLC-MS/ MS with positive ion mode electrospray ionization, one for analysis by UPLC-MS/MS with negative ion mode electrospray ionization, one for LC polar platform, and one sample was reserved for backup. For GC, each sample was dried under vacuum overnight before preparation for analysis as we previously described [14]. Likewise, the samples for LC were stored overnight under nitrogen followed by further LC analysis [14]. The metabolites present in those two groups of HCT-116 cells were identified using an automated comparison with Metabolon's reference library entries [14, 25, 26]. Library matching for each metabolite was performed for each sample and corrected manually if necessary. Furthermore to ensure the highest data quality for the metabolomic data analysis, a series of quality control and curation procedures were conducted.

\section{Data analysis}

For apoptosis analysis, each cell sample was analyzed in three replicates. Data are presented as means \pm standard deviations. A Welch independent sample $t$-test was performed to identify significant differences in apoptosis rates between the treated and untreated groups. Statistical analyses were performed using SPSS software (version 17.0; SPSS Institute, Chicago, IL). A $p$ value equals or less than 0.05 were considered statistically significant. After data normalization, the metabolomic data were analyzed as previously reported $[14,25,26]$. First, all the metabolomic data were subjected to Multi Experiment Viewer software (version 4.8; http://www.jcvi.org/cms/research/software/) for hierarchical clustering by using the Pearson correlation coefficient. Secondly, SIMCA-P software (version 13.0; MKS Data Analytics Solutions, Malmö, Sweden) was employed to perform partial least squares discriminant analysis (PLS-DA). In the PLSDA model, variables (metabolites) with differing importance in the project (VIP) values greater than 1 were selected for independent t-tests. Finally, variables (metabolites) with significant differences ( $p \leq 0.05)$ in the $\mathrm{t}$-test results were considered to be significantly different, which played a role on separation for the treated and untreated groups.

\section{Results}

$M B$ induces apoptosis of CRC cells

We initially found that HCT-116 CRC cells treated with MB showed decreased cell viability in a dose-dependent manner, with accompanying morphological changes (Fig. 1B-C). We then performed flow cytometry of HCT-116 cells to quantify apoptosis in the 
presence of $\mathrm{MB}$ treatment at concentrations of $20,50,100$, 200 , and $400 \mu \mathrm{M}$. MB induces significant apoptosis dosedependently at concentrations of $20,50,100,200$ and $400 \mu \mathrm{M}$ (Fig. 1D). Early apoptotic cell percentage was also calculated which showed that MB induced early apoptosis at all tested concentrations (Fig. 1D). To validate the apoptosis-inducing effects of $\mathrm{MB}$, immunoblot of caspase 3 and cleaved caspase 3 were determined in HCT-116 cells with $200 \mu \mathrm{M}$ MB treatment for 24 hours. The result showed that MB treatment altered the apoptosis markers, confirming the apoptosis-enhancing effect from MB (Fig. 1E).

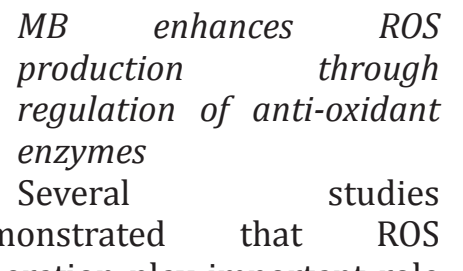
generation play important role in the induction of apoptosis under both physiologic and pathologic conditions $[3,4,9]$. To investigate whether apoptosis induced by MB is related with ROS generation, we measured ROS product using lucigenin derived chemiluminescence. MB markedly increased the ROS products (Fig. 2A). Meanwhile, we found the mRNA level of both MnSOD and GSH-Px were decreased in the MB-treated cells (Fig. 2B-C), suggesting that increased ROS generation may be associated with a decreased level of antioxidant enzymes.

\section{$M B$ represses xenograft tumor growth in vivo}

To investigate the antitumor activity of $M$ in vivo, four-week nude mice were injected with HCT-116, and then administrated with macranthoidin B or vehicle control through intraperitoneal injection respectively. Tumor volume was monitored every 7 days post carcinoma cell injection. Two weeks post tumor cell injection, mice were executed and tumor tissues were removed and weighted. The result showed that the tumor size and weight of tumors from MB group were significantly lower than the control group (Fig. 3A-C).

Lastly, immunohistochemistry of tumor tissues were performed to determine apoptosis 


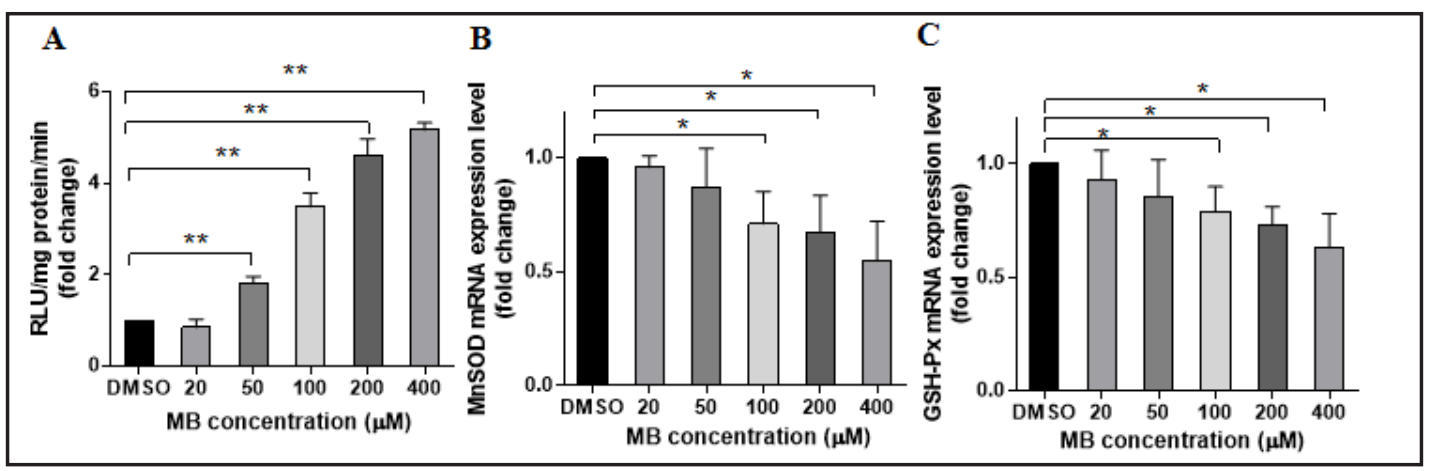

Fig. 2. MB induces ROS generation through regulation of anti-oxidant enzymes. (A) Dose-dependent decrease of ROS products with MB treatment at DMSO, 20, 50, 100, 200 and $400 \mu$ M concentrations in HCT-116 cells. (B) Dose-dependent decrease of ROS products with MB treatment at DMSO, 20, 50, 100, 200 and 400 $\mu \mathrm{M}$ concentrations in HCT-116 cells. (C) Dose-dependent increases of ROS products with MB treatment at DMSO, 20, 50, 100, 200 and $400 \mu \mathrm{M}$ concentrations in HCT-116 cells. ${ }^{*} \mathrm{P}<0.05,{ }^{* *} \mathrm{P}<0.01$.

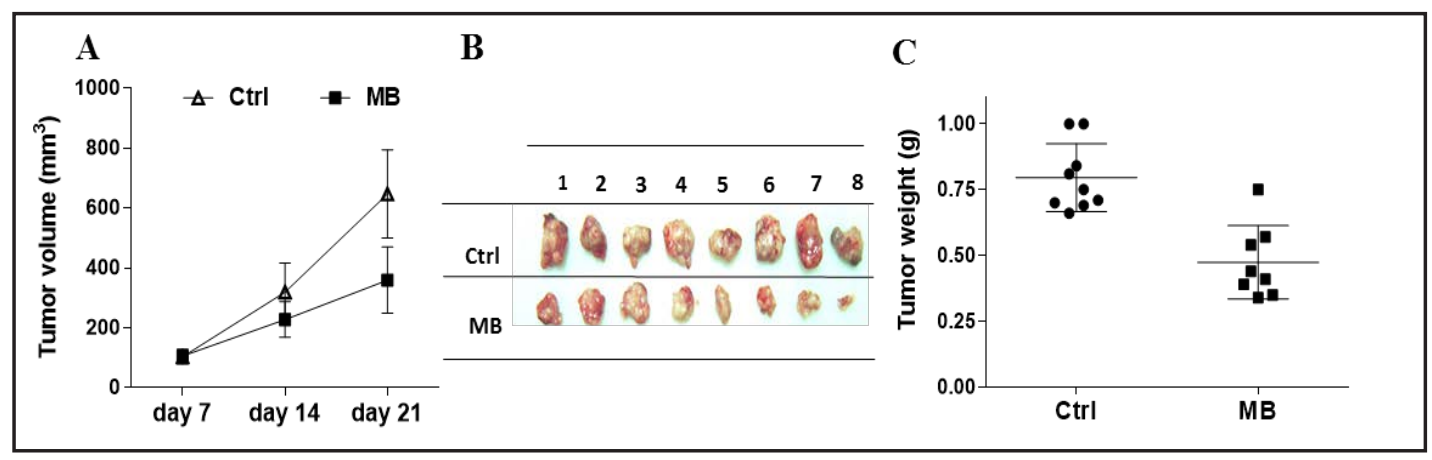

Fig. 3. MB represses CRC xenograft tumor growth in vivo. (A) Four-week-old nude mice were engrafted with HCT-116 cells and randomly divided into 2 groups. Tumor-bearing mice were then treated with vehicle and MB (16 mg/kg) ( $\mathrm{n}=8)$ by i.p. once a day for a total of $3 \mathrm{wk}$. Tumor volumes were calculated by the length and width measured by Vernier calipers every $7 \mathrm{~d}$. (B) Images of tumors from (A). (C) Tumor weight from (B).

by TUNEL and cleaved caspase-3. Few TUNEL staining was observed in control mice while MB-treated mice exhibited significantly increasing level of TUNEL positive cells per field (Fig. 4A-B) in CRC tissues. Cleaved caspase-3 level in control mice was lower compared with MB treatment mice (Fig. 5A-B), suggesting MB induces apoptosis. Taken together, these results demonstrate that MB-mediated tumor growth inhibition is associated with apoptosis.

\section{Metabolic characterization of HCT-116 cells}

In this study, by taking advantage of an established global metabolic profiling approach that combined GC-MS with UPLC/MS-MS [14], we comprehensively uncovered the metabolic profiles of HCT-116 cells with MB exposure. Totally 236 named metabolites were identified in HCT-116 cells before and after MB treatment. These metabolites were mapped to all the eight central pathways including 60 amino acids, 25 carbohydrates, 15 co-factors or vitamins, 81 lipids, 24 nucleotides, 16 peptides, 7 xenobiotics, and 8 metabolites involved in energy metabolism. As compared with previous studies, these findings uncover a much broader metabolome for HCT-116 cells and provide abundant data for the determination of metabolic alteration induced by MB $[14,27,28]$.

\section{Metabolic alteration in HCT-116 cells after MB exposure}

To review the similarities and differences of the identified metabolites between MB -treated and untreated HCT-116 cells, hierarchical cluster analysis was employed for all samples (Fig. 6). The heat map revealed remarkable variation between two groups, each 
Fig. 4. MB represses colorectal carcinoma xenograft tumor growth in vivo. (A) TUNEL positive cells were observed in different groups (40x, scale bar $=100 \mu \mathrm{m}$ ), black arrows indicated TUNEL positive cells, (B) Analysis of the TUNEL positive cells per field in each group. Compared with the Ctrl group, the mean number of TUNEL positive cells per field was significantly in-

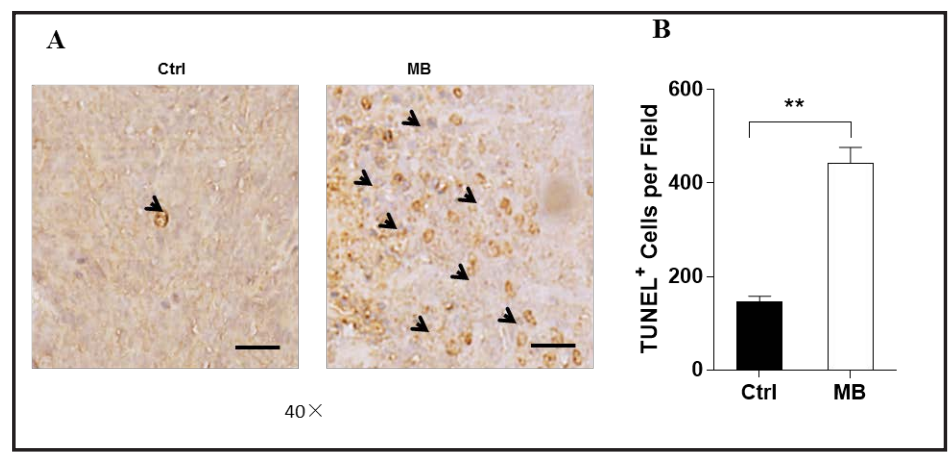
creased in the MB group. Values are means $\pm \operatorname{SD}\left(n=6\right.$ per group). ${ }^{*} \mathrm{P}<0.05,{ }^{* *} \mathrm{P}<0.01$.

Fig. 5. MB represses colorectal carcinoma xenograft tumor growth in vivo. (A) Cleaved caspase-3 cells were observed in different groups (40x, scale bar $=100 \mu \mathrm{m})$, black arrows indicated cleaved caspase-3 cells, (B) Analysis of the cleaved caspase-3 cells per field in each group. Compared with the Ctrl group, the mean number of cleaved

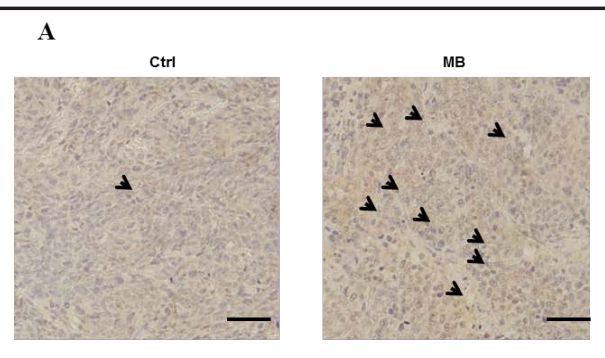

$40 \times$
$\mathbf{B}$

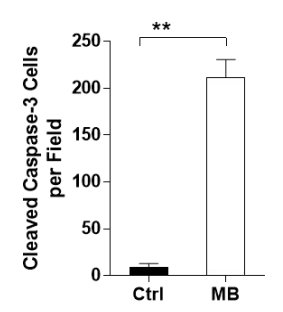
caspase-3 cells per field was significantly increased in the MB group. Values are means $\pm \mathrm{SD}\left(n=6\right.$ per group). ${ }^{*} \mathrm{P}<0.05,{ }^{* *} \mathrm{P}<0.01$.

contained 4 replicates. Intriguingly, the 236 identified metabolites differed markedly between the MB-treated and untreated groups. In most cases, MB reduces the metabolites that were highly abundant in untreated HCT-116 cells and increases the metabolites that were at low levels in untreated cells. For example, the levels of ethanolamine in the MBtreated group were higher than those in the untreated group, but S-adenosylhomocysteine $(\mathrm{SAH})$ was more abundant in the untreated group than in the MB-treated group.

To further determine the metabolic alteration in HCT-116 cells induced by MB treatment, a supervised statistical method of PLS-DA was employed. PLS-DA is a powerful method for compositional (metabolic) data that contains more metabolites (in hundreds) than biological materials (only tens) [29]. The results showed that levels of 31 metabolites (Table 1; Fig. 7A-B) were significantly different between the two groups $(p \leq 0.05)$. Meanwhile, these 31 metabolites included 21 increased and 10 decreased metabolites covered all the eight major pathways, with the magnitude of the changes ranging from 0.35 - to 4.95 -fold. As shown in Table 1, the concentrations of 6 nucleotides, 2 amino acids, 1 peptide, and 1 lipid were decreased after MB exposure. Meanwhile, the levels of 3 co-factors or vitamins, 4 carbohydrates, 2 amino acids, 2 peptides, 7 lipids and citrate were increased after the treatment. The results here suggest remarkable metabolic alteration involved in colorectal cell metabolism including amino acid metabolism, carbohydrate metabolism, and lipid metabolism.

\section{Metabolic network in MB-treated cells}

To fully reveal the regulatory network of 31 changed metabolites in MB-treated cells, we build up a metabolic network for amino acids and peptides, carbohydrates, lipids, nucleotides, cofactors and vitamins metabolism and xenobiotics. As shown in Fig. 8, 5 metabolites (glucose, fructose, 3-phosphoglycerate, 2-phosphoglycerate, and citrate) in carbohydrate metabolism displayed elevation in MB-treated HCT-116 cells. While in the metabolism of amino acid and peptide, the levels of four amino acids and three peptides displayed significantly 
Fan et al.: Macranthoidin B Modulates Key Metabolic Pathways in Colorectal Cancer

Fig. 6. Heat map representation of 236 metabolites between treated and untreated groups in clustering analysis. Each line represented a metabolite. Deeper red color represents higher content in the cell lines; similarly, deeper green color represents lower content in the cell lines. Three metabolites mentioned in the text are shown in greater detail by boxplot. The $p$ values were calculated between the MB-treated and untreated groups.

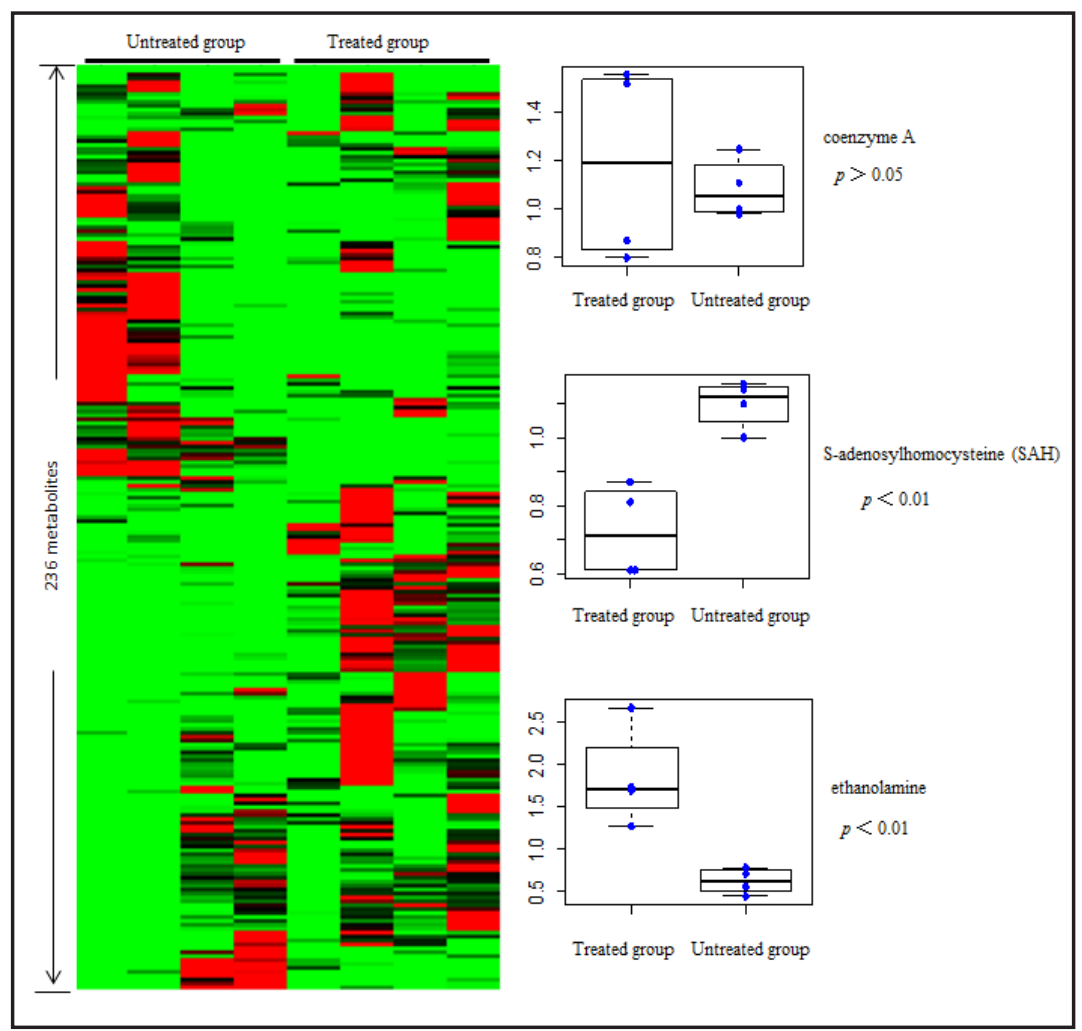

changed. Among them, $\gamma$ glut a m ylglut a mate, 2-aminobutyrate, and arginine associated with glutamate were included but glutamate was determined to be unchanged. Meanwhile, lower level of $\mathrm{S}$-adenosylhomocysteine (SAH) in macranthoidin B-treated HCT-116 cells was observed. Enhanced lipid metabolism is now recognized as one of the key features of cancer cells. Here levels of 7 lipids were significantly increased after MB treatment, while the level of 1-palmitoylglycerol (16:0) was conversely decreased. Those increased metabolites were ethanolamine, 1-stearoylGPE (18:0), 1-palmitoylGPC (16:0), sphinganine, cholestanol, 1-oleoyl-GPG (18:1), and sphinganine. Moreover, levels of all 6 metabolites involved in
Table 1. Metabolites determined at significantly different levels in MBtreated and untreated HCT-116 colorectal cancer cells. *represented fold change between treated and untreated group. GPC: glycerophosphocholine; GPE: glycerophosphoethanolamine; GPG: phosphoglycerol; SAH: S-adenosylhomocysteine; AMP: adenosine 5'-monophosphate; IMP: inosine 5'-monophosphate; 5'-GMP: guanosine 5'-monophosphate; 5'-CMP: cytidine 5'-monophosphate; NaMN: nicotinic acid mononucleotide

\begin{tabular}{lccc}
\hline Super pathway & Biochemical & Fold change* & p value \\
\hline \multirow{4}{*}{ Amino Acid } & S-adenosylhomocysteine (SAH) & 0.66 & $5.70 \mathrm{E}-03$ \\
& 2-aminobutyrate & 2.25 & $1.60 \mathrm{E}-03$ \\
& phenylalanine & 1.39 & $2.24 \mathrm{E}-02$ \\
& arginine & 0.62 & $4.16 \mathrm{E}-02$ \\
Carbohydrate & fructose & 4.95 & $1.03 \mathrm{E}-02$ \\
& glucose & 1.94 & $4.55 \mathrm{E}-02$ \\
& 3-phosphoglycerate & 2.32 & $4.13 \mathrm{E}-02$ \\
Cofactors and Vitamins & 2-phosphoglycerate & 3.69 & $3.53 \mathrm{E}-02$ \\
& nicotinamide riboside* & 1.8 & $5.51 \mathrm{E}-03$ \\
Energy & nicotinic acid mononucleotide (NaMN) & 2.5 & $1.03 \mathrm{E}-02$ \\
& nicotinate ribonucleoside* & 3.04 & $1.31 \mathrm{E}-03$ \\
& citrate & 1.7 & $3.86 \mathrm{E}-02$ \\
& 1-oleoyl-GPG (18:1)* & 1.6 & $1.31 \mathrm{E}-02$ \\
Lipid & 1-stearoyl-GPE (18:0) & 1.63 & $1.01 \mathrm{E}-02$ \\
& 1-palmitoyl-GPC (16:0) & 2.18 & $1.79 \mathrm{E}-02$ \\
& 1-palmitoylglycerol (16:0) & 0.53 & $3.31 \mathrm{E}-02$ \\
& glycerophosphorylcholine (GPC) & 1.85 & $4.01 \mathrm{E}-03$ \\
& ethanolamine & 2.97 & $7.16 \mathrm{E}-03$ \\
& sphinganine & 1.64 & $8.86 \mathrm{E}-03$ \\
Nucleotide & cholestanol & 1.42 & $2.31 \mathrm{E}-02$ \\
& inosine 5'-monophosphate (IMP) & 0.58 & $9.19 \mathrm{E}-04$ \\
& adenosine 5'-monophosphate (AMP) & 0.62 & $7.90 \mathrm{E}-03$ \\
Peptide & adenine & 0.62 & $5.50 \mathrm{E}-03$ \\
& guanosine 5'- monophosphate (5'-GMP) & 0.71 & $6.00 \mathrm{E}-03$ \\
Xenobiotics & cytidine 5'-monophosphate (5'-CMP) & 0.49 & $2.15 \mathrm{E}-05$ \\
& pseudouridine & 0.35 & $6.26 \mathrm{E}-03$ \\
& valylleucine & 1.44 & $3.63 \mathrm{E}-02$ \\
& glycylisoleucine & 2.13 & $1.70 \mathrm{E}-02$ \\
& gama-glutamylglutamate & 0.46 & $1.53 \mathrm{E}-02$ \\
& phenol red & 1.49 & $2.48 \mathrm{E}-03$ \\
& erythritol & 1.62 & $1.50 \mathrm{E}-02$ \\
\hline & & &
\end{tabular}


Fig. 7. Metabolites determined at significantly different levels between MB-treated and untreated HCT-116 cells. (A) Plot of the scores from the PLS-DA model of the treated and untreated groups. (B) Loading plot from the PLS-DA model of the treated and untreated groups. The boxes represent the tested samples while metabolites are displayed as triangles. Metabolites playing key roles for separation are marked with red triangle.
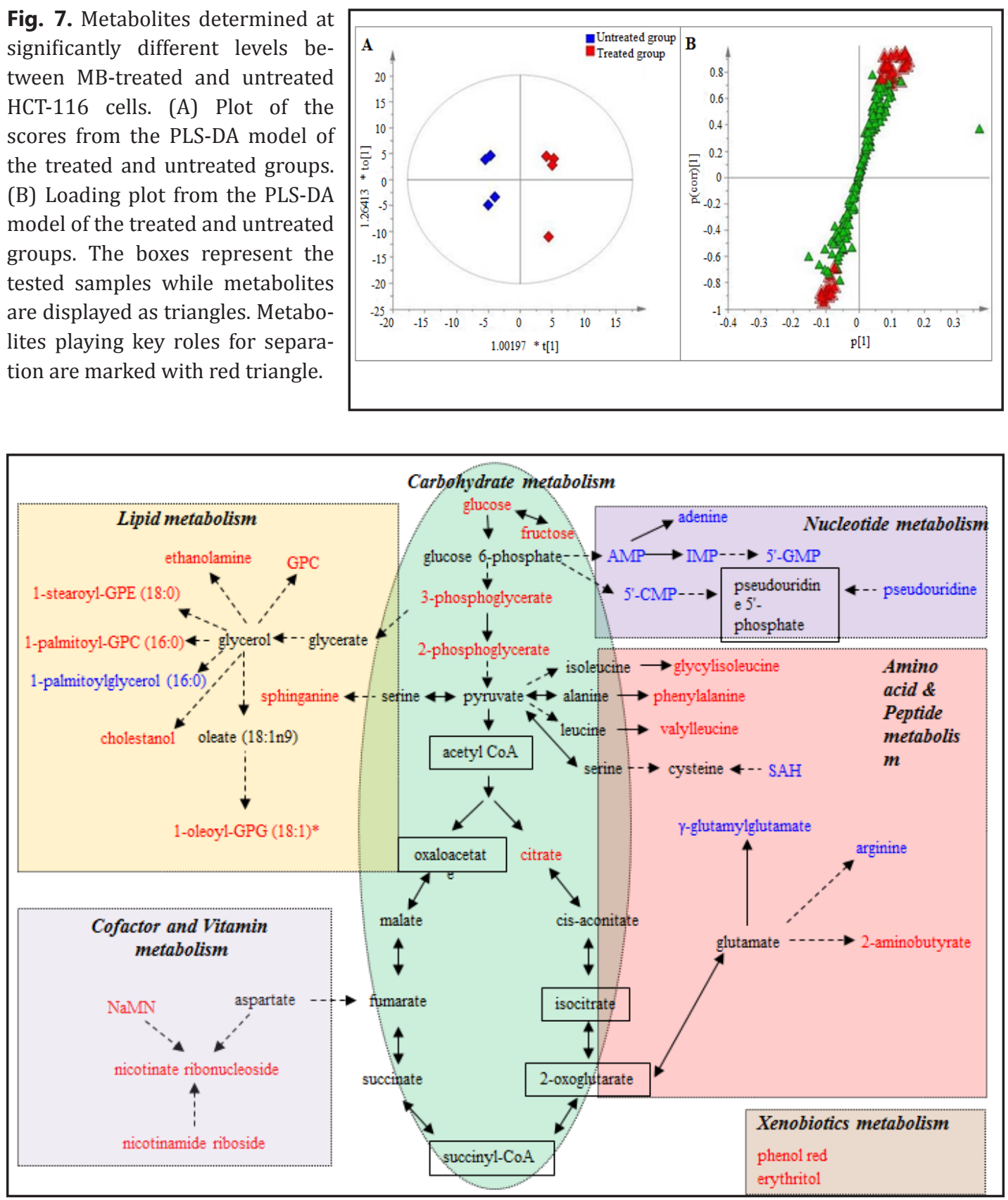

Fig. 8. Metabolic networks involving metabolites that were significantly increased or decreased in MBtreated HCT-116 cells. Metabolites undetected in both the treated and untreated groups are shown in the black boxes. Metabolites in red or blue exhibited increased or decreased levels, respectively, in the macranthoidin B-treated group. GPC: glycerophosphocholine; GPE: glycerophosphoethanolamine; GPG: phosphoglycerol; SAH: S-adenosylhomocysteine; AMP: adenosine 5'-monophosphate; IMP: inosine 5'-monophosphate; 5'-GMP: guanosine 5'-monophosphate; 5'-CMP: cytidine 5'-monophosphate; NaMN: nicotinic acid mononucleotide.

nucleotide metabolism were significantly decreased, which included AMP, IMP, 5'-GMP, 5'-CMP, pseudouridine, and adenine. In addition, there were 5 significantly increased metabolites participated in cofactor and vitamin metabolism, and xenobiotics metabolism: NaMN, nicotinate ribonucleoside, nicotinamide riboside, phenol red, and erythritol. 


\section{Cellular Physiology Cell Physiol Biochem 2018;46:1317-1330 \\ \begin{tabular}{ll|l} 
DOI: 10.1159/000489147 & C 2018 The Author(s). Published by S. Karger AG, Basel \\
www.karger.com/cpb
\end{tabular}}

Fan et al.: Macranthoidin B Modulates Key Metabolic Pathways in Colorectal Cancer

\section{Discussion}

To comprehensively elucidate the anti-tumor mechanism of MB, here for the first time we employed an established non-targeted metabolomic analysis platform that combined UPLC/MS-MS with GC-MS to profile HCT-116 cells treated with MB. Totally 236 known metabolites were determined in HCT-116 cells, which were mapped to most of the central metabolism pathways including amino acid super pathway, carbohydrate super pathway, lipid super pathway, and nucleotide super pathway. The results thus far uncover a much broader metabolome, manifesting the power of this untargeted metabolomic profiling platform in elucidating metabolic characterization of HCT-116 cells [14, 27, 28]. More importantly, significant metabolic alterations in HCT-116 cells after MB treatment were identified, including changes of several important metabolites and related pathways. These metabolic changes in response to MB may play important roles in repressing tumor progression and inducing apoptosis in CRC cells. The results here provide new insights into metabolic changes and regulatory aspects of the metabolic pathways associated with antitumor mechanism of MB.

Cancer cells usually exhibit an altered metabolic pattern to support the proliferation and tumor growth [30]. In our study, several important metabolites and key metabolic pathways were modulated in HCT-116 cells with MB exposure. For example, cancer cells generally exhibit increased glycolysis for ATP generation (the Warburg effect) due in part to mitochondrial respiration injury and hypoxia, which are frequently associated with resistance to therapeutic agents [31]. In line with the literature, significant alteration of some of the glycolytic intermediates was noted in cells treated with MB compared to untreated control cells, which may be an indication of increased glucose uptake and potential alteration in glycolysis within the cells treated with MB [14]. Moreover, a significant increase in most of the glycolytic intermediates including glucose, fructose, citrate, 3-phosphoglycerate and 2-phosphoglycerate was displayed in cells treated with MB, suggesting alteration in glycolytic energy metabolism in cancer cells by MB. Especially, the convert of 3-phosphoglycerate to 2-phosphoglycerate (2-PG), catalyzed by phosphoglycerate mutase (PGAM), is commonly upregulated in many human cancers and plays an important role in cancer metabolism [32]. Besides, increased glutamine metabolism is another commonly metabolic alteration observed in cancer cells, supporting cell growth [14]. Although both the concentrations of glutamine and glutamate did not change, the level of one related peptide, $\gamma$-glutamylglutamate showed significantly decreased. Furthermore, HCT-116 cells treated with MB exhibited significant changes of other 6 metabolites (arginine, phenylalanine, and SAH) involved in the metabolism of amino acid and peptide, which plays important roles in colorectal cancer development and progression. For example, the level of arginine in MB-treated HCT116 cells was significantly decreased, which probably resulted from the treatment by MB. Accumulating evidence has shown the contributions made to inhibit this pathway for cancer treatment, since overactive arginine catabolism may be a common phenomenon in cancer development and progression [33]. As reported, arginine is synthesized from glutamate via the intestinal-renal axis in humans but glutamate was determined to be unchanged. Notably, the decrease in SAH levels identified in MB-treated cells may indicate the inhibition of CRC cell proliferation, since accumulation of SAH in body fluids was reported to be associated with vascular disease and CRC $[34,35]$. The level of three metabolites related to pyruvate was all upregulated in MB-treated HCT-116 cells including phenylalanine. High level of phenylalanine may indicate a higher protein degradation rate [36]. The other increased metabolite was 2-aminobutyrate, which was determined to be one of specific bioindicators in colorectal cancer patients [37]. Likewise, remarkable metabolic changes related to lipid metabolism were also observed, including 8 lipids and their corresponding metabolic pathways. Among them, sphinganine may potentiate apoptosis in HCT-116 cells after MB exposure [38]. Likewise, cholestanol was reported with CRC progression via activation of ROS [39], and high level of cholestanol might be associated with apoptosis in HCT-116 cells, 


\section{Cellular Physiology Cell Physiol Biochem 2018;46:1317-1330

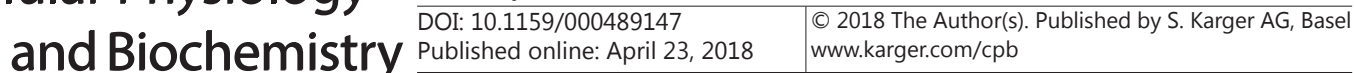 \\ Fan et al.: Macranthoidin B Modulates Key Metabolic Pathways in Colorectal Cancer}

since it is a marker of cholesterol absorption, which was reported to cause endoplasmic reticulum stress-induced apoptosis [40]. The results here also revealed an alteration of nucleotide metabolism associated with AMP, IMP, 5'-GMP, 5'-CMP, and pseudouridine, which are necessary for a number of key cellular processes, and imbalance in their levels lead to tumor progression [41]. AMPK is a key regulator of metabolism and can negatively regulate tumor proliferation, which functions as a cellular energy sensor primarily regulated by AMP [42]. Another nucleotide, pseudouridine, was reported to be a putative marker in various types of cancer and heart failure [43]. Additionally, several cofactors and vitamins including nicotinamide riboside, $\mathrm{NaMN}$, and nicotinate ribonucleoside were accumulated in MB-treated cells. These metabolites were participated in the NAD metabolome that was reported to be a key determinant of cancer cell growth. High level of nicotinamide riboside, the $\mathrm{NAD}^{+}$precursor may lead to more active oxidative metabolism in MB-treated HCT-116 cells [44]. Taken together, the metabolic alterations in treated cells indicate an important role of MB in suppressing CRC cell proliferation, inducing apoptosis, and inhibiting tumor progression. It was further validated in in vivo experiments that MB can actually reduce CRC xenograft tumor growth.

Apoptosis is deregulated in multiple cancer types, making eradication of cancer cells a difficult challenge [45]. Consequently, anti-cancer agents or other therapies that promote apoptosis are under development as alternative strategy in cancer therapy discovery. To date, most of phytochemical compounds originated from TCM have been identified based on their activity on inducing apoptosis in cancer cells [46-48]. For example, Berberine, a wellknown TCM compound initially extracted from the herb Huanglian (Coptis chinensis) has been shown to induce ROS-mediated apoptosis in various cancers [49]. Levistolide A (LA), a natural compound isolated from the traditional Chinese herb Ligusticum chuanxiong Hort, was found induce apoptosis of colon cancer cells via ROS-mediated ER stress pathway [50]. In present study, MB, also isolated from the Flos Lonicerae (Shanyinhua in Chinese), was demonstrated to promote apoptosis in a dose-dependent manner. In addition to modulate glucose and glutamine metabolism, MB was found to significantly impact several important apoptosis-related metabolites such as sphinganine and cholestanol in HCT-116 cells [51]. Generation of ROS plays an important role in the mitochondria-mediated intrinsic apoptotic pathway, which is regulated by oxidative stress response mechanism [52]. Our study showed a significant decrease of SAH level in CRC cells treated with MB suggests an enhanced generation of ROS, since SAH functions as a major cellular anti-oxidant [53]. Moreover, level of $\gamma$-glutamylglutamate was decreased, reflecting the changes associated with a key survival antioxidant, glutathione. It is believed that caspase- 3 is the primary executioner of apoptosis [54]. As a result, we also evaluated alteration of caspase-3 protein, and we found high levels of caspase- 3 in MB-treated CRC cells. These results suggest that MB induces apoptosis in HCT-116 cells by promoting the generation of ROS and the activation of caspase- 3 .

In the present study, we, for the first time, revealed the anti-tumor mechanism of MB, extracted from the Flos Lonicerae. Our results show remarkable metabolic alterations of differential metabolite production and activation of metabolic pathways in HCT-116 CRC cells with MB treatment, eventually leading to induced apoptosis through enhanced ROS production and activated caspase-3. This study provides new insights into of the mechanisms by which MB suppresses CRC growth and progression, supporting its potential implication in future development for treating CRC. We are currently identifying whether there is mitochondrial dysfunction including the determination of mitochondrial membrane potential and ROS generation. The expression of apoptosis-related genes such as Bax and Bcl-2 are also under investigation in HCT-116 CRC cells with MB treatment. Moreover, proteomics and transcriptomics analyses are to be employed to explore and validate the anti-tumor mechanisms of MB, in addition to evaluating the usefulness of MB as a novel anticancer therapy for CRC and other cancer types. 


\section{Cellular Physiology Cell Physiol Biochem 2018;46:1317-1330 \begin{tabular}{l|l} 
DOI: 10.1159/000489147 & $\begin{array}{l}\text { O } 2018 \text { The Author(s). Published by S. Karger AG, Basel } \\
\text { www.karger.com/cpb }\end{array}$
\end{tabular}}

\section{Acknowledgements}

This work was supported by grant No. 81260592 and No. 81303119 from the National Natural Science Foundation of China; NO. 20171BBG70119 and 20171BAB215068 from the key research and development plan of Jiangxi provincial department of science; in part by a Cancer Center Support Grant CA016672 from the NIH/NCI (MDACC); Startup fund from MDACC (QS); and Duncan Family Institute Seed Funding Research Program (QS).

\section{Disclosure Statement}

The authors declare no conflicts of interest.

\section{References}

$>1$ Devasagayam TP, Tilak JC, Boloor KK, Sane KS, Ghaskadbi SS, Lele RD: Free radicals and antioxidants in human health: current status and future prospects. J Assoc Physicians India 2004;52:794-804.

2 Moloney JN, Cotter TG: ROS signalling in the biology of cancer. Semin Cell Dev Biol 2017;10.1016/j. semcdb.2017.05.023

3 Renschler MF: The emerging role of reactive oxygen species in cancer therapy. Eur J Cancer 2004;40:19341940.

4 Trachootham D, Alexandre J, Huang P: Targeting cancer cells by ROS-mediated mechanisms: a radical therapeutic approach? Nat Rev Drug Discov 2009;8:579-591.

-5 Chovancova B, Hudecova S, Lencesova L, Babula P, Rezuchova I, Penesova A, Grman M, Moravcik R, Zeman M, Krizanova O: Melatonin-Induced Changes in Cytosolic Calcium Might be Responsible for Apoptosis Induction in Tumour Cells. Cell Physiol Biochem 2017;44:763-777.

6 Toler SM, Noe D, Sharma A: Selective enhancement of cellular oxidative stress by chloroquine: implications for the treatment of glioblastoma multiforme. Neurosurg Focus 2006;21:E10.

7 Izuishi K, Kato K, Ogura T, Kinoshita T, Esumi H: Remarkable tolerance of tumor cells to nutrient deprivation: possible new biochemical target for cancer therapy. Cancer Res 2000;60:6201-6207.

-8 Storz P: Reactive oxygen species in tumor progression. Front Biosci 2005;10:1881-1896.

-9 Idelchik M, Begley U, Begley TJ, Melendez JA: Mitochondrial ROS control of cancer. Semin Cancer Biol 2017;10.1016/j.semcancer.2017.04.005

10 Shen B: A New Golden Age of Natural Products Drug Discovery. Cell 2015;163:1297-1300.

11 Ma HD, Deng YR, Tian Z, Lian ZX: Traditional Chinese medicine and immune regulation. Clin Rev Allergy Immunol 2013;44:229-241.

-12 Ling CQ, Yue XQ Ling C: Three advantages of using traditional Chinese medicine to prevent and treat tumor. J Integr Med 2014;12:331-335.

-13 Ji Y, Rao J, Rong X, Lou S, Zheng Z, Lu Y: Metabolic characterization of human aqueous humor in relation to high myopia. Exp Eye Res 2017;159:147-155.

14 Zheng Z, Xu L, Zhang S, Li W, Tou F, He Q, Rao J, Shen Q: Peiminine inhibits colorectal cancer cell proliferation by inducing apoptosis and autophagy and modulating key metabolic pathways. Oncotarget 2017;8:47619-47631.

15 Wang X, Sun H, Zhang A, Sun W, Wang P, Wang Z: Potential role of metabolomics apporoaches in the area of traditional Chinese medicine: as pillars of the bridge between Chinese and Western medicine. J Pharm Biomed Anal 2011;55:859-868.

16 Martin FP, Collino S, Rezzi S, Kochhar S: Metabolomic applications to decipher gut microbial metabolic influence in health and disease. Front Physiol 2012;3:113.

17 Raina K, Agarwal C, Wadhwa R, Serkova NJ, Agarwal R: Energy deprivation by silibinin in colorectal cancer cells: a double-edged sword targeting both apoptotic and autophagic machineries. Autophagy 2013;9:697713. 


\section{Cellular Physiology Cell Physiol Biochem 2018;46:1317-1330 \begin{tabular}{l|l} 
DOI: 10.1159/000489147 & Ond Biochemistry 2018 The Author(s). Published by S. Karger AG, Basel \\
wwww.karger.com/cpb
\end{tabular}}

Fan et al.: Macranthoidin B Modulates Key Metabolic Pathways in Colorectal Cancer

18 Cui DN, Wang X, Chen JQ, Lv B, Zhang P, Zhang W, Zhang ZJ, Xu FG: Quantitative Evaluation of the Compatibility Effects of Huangqin Decoction on the Treatment of Irinotecan-Induced Gastrointestinal Toxicity Using Untargeted Metabolomics. Front Pharmacol 2017;8:211.

19 China, Pharmacopoeia C: Pharmacopoeia of the People's Republic of China, China Medical Science Press, Beijing, 2010.

20 Chai XY, Li SL, Li P: Quality evaluation of Flos lonicerae through a simultaneous determination of seven saponins by HPLC with ELSD. J Chromatogr A 2005;1070:43-48.

-21 Chen CY, Qi LW, Yi L, Li P, Wen XD: Liquid chromatography-mass spectrometry analysis of macranthoidin B, macranthoidin A, dipsacoside B, and macranthoside B in rat plasma for the pharmacokinetic investigation. J Chromatogr B Analyt Technol Biomed Life Sci 2009;877:159-165.

22 Jiang P, Sheng YC, Chen YH, Ji LL, Wang ZT: Protection of Flos Lonicerae against acetaminophen-induced liver injury and its mechanism. Environ Toxicol Pharmacol 2014;38:991-999.

23 Ohta S, Sato N, Tu SH, Shinoda M: [Protective effects of Taiwan crude drugs on experimental liver injuries]. Yakugaku Zasshi 1993;113:870-880.

-24 Cui W, Matsuno K, Iwata K, Ibi M, Matsumoto M, Zhang J, Zhu K, Katsuyama M, Torok NJ, Yabe-Nishimura C: NOX1/nicotinamide adenine dinucleotide phosphate, reduced form (NADPH) oxidase promotes proliferation of stellate cells and aggravates liver fibrosis induced by bile duct ligation. Hepatology 2011;54:949-958.

25 Halama A, Guerrouahen BS, Pasquier J, Diboun I, Karoly ED, Suhre K, Rafii A: Metabolic signatures differentiate ovarian from colon cancer cell lines. J Transl Med 2015;13:223.

26 Rao J, Cheng F, Hu C, Quan S, Lin H, Wang J, Chen G, Zhao X, Alexander D, Guo L, Wang G, Lai J, Zhang D, Shi J: Metabolic map of mature maize kernels. Metabolomics 2014;10:775-787.

27 Gao D, Wang Y, Xie W, Yang T, Jiang Y, Guo Y, Guan J, Liu H: Metabolomics study on the antitumor effect of marine natural compound flexibilide in HCT-116 colon cancer cell line. J Chromatogr B Analyt Technol Biomed Life Sci 2016;1014:17-23.

28 Tolstikov V, Nikolayev A, Dong S, Zhao G, Kuo MS: Metabolomics analysis of metabolic effects of nicotinamide phosphoribosyltransferase (NAMPT) inhibition on human cancer cells. PLoS One 2014;9:e114019.

29 Kalivodová A, Hron K, Filzmoser P, Najdekr L, Janečková H, Adam T: PLS-DA for compositional data with application to metabolomics. J Chemomet 2015;29:21-28.

-30 Sullivan LB, Gui DY, Heiden MGV: Altered metabolite levels in cancer: implications for tumour biology and cancer therapy. Nat Rev Cancer 2016;16:680-693.

31 Dang CV: Links between metabolism and cancer. Genes \& Development 2012;26:877-890.

-32 Hitosugi T, Zhou L, Fan J, Elf S, Zhang L, Xie JX, Wang Y, Gu TL, Aleckovic M, LeRoy G, Kang YB, Kang HB, Seo JH, Shan CL, Jin P, Gong WM, Lonial S, Arellano ML, Khoury HJ, Chen GZ, Shin DM, Khuri FR, Boggon TJ, Kang SM, He C, Chen J: Tyr26 phosphorylation of PGAM1 provides a metabolic advantage to tumours by stabilizing the active conformation. Nat Commun 2013;4:

33 Chen B, Wang W, Ding K, Wang J, Gao P, Xu G, Hu H: l-Arginine and Its Transporters in Colorectal Cancer; in (Patel VB, Preedy VR, and Rajendram R, eds) L-Arginine in Clinical Nutrition. Cham, Springer International Publishing, 2017, vol. 10.1007/978-3-319-26009-9_19p.^pp. 239-252.

34 DeBerardinis RJ, Lum JJ, Hatzivassiliou G, Thompson CB: The biology of cancer: metabolic reprogramming fuels cell growth and proliferation. Cell Metab 2008;7:11-20.

35 Valli A, Carrero JJ, Qureshi AR, Garibotto G, Barany P, Axelsson J, Lindholm B, Stenvinkel P, Anderstam B, Suliman ME: Elevated serum levels of S-adenosylhomocysteine, but not homocysteine, are associated with cardiovascular disease in stage 5 chronic kidney disease patients. Clin Chim Acta 2008;395:106-110.

-36 Jimenez B, Mirnezami R, Kinross J, Cloarec O, Keun HC, Holmes E, Goldin RD, Ziprin P, Darzi A, Nicholson JK: 1H HR-MAS NMR spectroscopy of tumor-induced local metabolic "field-effects" enables colorectal cancer staging and prognostication. J Proteome Res 2013;12:959-968.

-37 Cheng Y, Xie GX, Chen TL, Qiu YP, Zou X, Zheng MH, Tan BB, Feng B, Dong TT, He PA, Zhao LJ, Zhao AH, Xu LX, Zhang Y, Jia W: Distinct Urinary Metabolic Profile of Human Colorectal Cancer. J Proteome Res 2012;11:1354-1363.

-38 Farhat M, Poissonnier A, Hamze A, Ouk-Martin C, Brion JD, Alami M, Feuillard J, Jayat-Vignoles C: Reversion of apoptotic resistance of TP53-mutated Burkitt lymphoma B-cells to spindle poisons by exogenous activation of JNK and p38 MAP kinases. Cell Death Dis 2014;5: 


\section{Cellular Physiology Cell Physiol Biochem 2018;46:1317-1330 and Biochemistry Published online: April 23, $2018 \quad \begin{aligned} & \text { DOI 10159/2018 The Author(s). Published by S. Karger AG, Basel } \\ & \text { www.karger.com/cpb }\end{aligned}$}

Fan et al.: Macranthoidin B Modulates Key Metabolic Pathways in Colorectal Cancer

39 Wang C, Li P, Xuan J, Zhu C, Liu J, Shan L, Du Q, Ren Y, Ye J: Cholesterol Enhances Colorectal Cancer Progression via ROS Elevation and MAPK Signaling Pathway Activation. Cell Physiol Biochem 2017;42:729742.

40 Beloribi-Djefaflia S, Vasseur S, Guillaumond F: Lipid metabolic reprogramming in cancer cells. Oncogenesis 2016;5:

41 Aird KM, Zhang RG: Nucleotide metabolism, oncogene-induced senescence and cancer. Cancer Lett 2015;356:204-210.

-42 Gowans GJ, Hardie DG: AMPK: a cellular energy sensor primarily regulated by AMP. Biochem Soc Transact2014;42:71-75.

43 Struck-Lewicka W, Kaliszan R, Markuszewski MJ: Analysis of urinary nucleosides as potential cancer markers determined using LC-MS technique. J Pharma Biomed Anal 2014;101:50-57.

44 Canto C, Houtkooper RH, Pirinen E, Youn DY, Oosterveer MH, Cen Y, Fernandez-Marcos PJ, Yamamoto H, Andreux PA, Cettour-Rose P, Gademann K, Rinsch C, Schoonjans K, Sauve AA, Auwerx J: The NAD(+) Precursor Nicotinamide Riboside Enhances Oxidative Metabolism and Protects against High-Fat DietInduced Obesity. Cell Metabolism 2012;15:838-847.

-45 Fesik SW: Promoting apoptosis as a strategy for cancer drug discovery. Nat Rev Cancer 2005;5:876-885.

46 Li-Weber M: Targeting apoptosis pathways in cancer by Chinese medicine. Cancer Lett2013;332:304-312.

47 Zhang YS, Shen Q, Li J: Traditional Chinese medicine targeting apoptotic mechanisms for esophageal cancer therapy. Acta Pharmacol Sin 2016;37:295-302.

-48 Kuo HC, Kuo YR, Lee KF, Hsieh MC, Huang CY, Hsieh YY, Lee KC, Kuo HL, Lee LY, Chen WP, Chen CC, Tung SY: A Comparative Proteomic Analysis of Erinacine A's Inhibition of Gastric Cancer Cell Viability and Invasiveness. Cell Physiol Biochem 2017;43:195-208.

-49 Meeran SM, Katiyar S, Katiyar SK: Berberine-induced apoptosis in human prostate cancer cells is initiated by reactive oxygen species generation. Toxicol Appl Pharmacol 2008;229:33-43.

50 Yang Y, Zhang Y, Wang L, Lee S: Levistolide A Induces Apoptosis via ROS-Mediated ER Stress Pathway in Colon Cancer Cells. Cell Physiol Biochem 2017;42:929-938.

51 Bruchelt G, Handgretinger R, Weckenmann M, Hahn T: Glucose Metabolism and the Antioxidative Defense System in Cancer Cells: Options for the Application of ROS-based Anticancer Drugs; in (Kanner S, ed) Tumor Metabolome Targeting and Drug Development. New York, NY, Springer New York, 2014, vol. 10.1007/978-1-4614-9545-1_5p.^pp. 109-130.

-52 Wang L, Hu T, Shen J, Zhang L, Li LF, Chan RLY, Li MX, Wu WKK, Cho CH: Miltirone induced mitochondrial dysfunction and ROS-dependent apoptosis in colon cancer cells. Life Sci 2016;151:224-234.

53 Lu SC: S-Adenosylmethionine. Int J Biochem Cell Biol 2000;32:391-395.

54 Brentnall M, Rodriguez-Menocal L, De Guevara RL, Cepero E, Boise LH: Caspase-9, caspase-3 and caspase-7 have distinct roles during intrinsic apoptosis. BMC Cell Biol 2013;14:32. 